\title{
DISTRIBUIÇÃO DIAMÉTRICA DE UM FRAGMENTO DE FLORESTA ATLÂNTICA NO MUNICÍPIO DE SANTA MARIA DE JETIBÁ, ES
}

\author{
José Imaña-Encinas ${ }^{1}$, Cláudio de Almeida Conceiçãoº ${ }^{2}$ Otacílio Antunes Santana ${ }^{3}$, \\ Christian Rainier Imaña ${ }^{4}$, José Elias de Paula ${ }^{5}$ \\ ${ }^{1}$ Eng. Florestal, Ph.D, Depto. Engenharia Florestal, UnB,Brasília, DF, Brasil - imana @unb.br \\ ${ }^{2}$ Biólogo, Depto. Botânica, UFMS, Campo Grande, MS, Brasil - claudioalmeidac@gmail.com \\ ${ }^{3}$ Biólogo, Dr., Centro Biologia, UFPE, Recife, PE, Brasil - otaciliosantana@ gmail.com \\ ${ }^{4}$ Estatístico, Auditor Fiscal, Secretaria da Fazenda do Estado de Minas Gerais, MG, Brasil - christian_bsb@hotmail.com \\ ${ }^{5}$ Botânico, Dr., Depto. Botânica, UnB, Brasília, DF, Brasil - depaula@unb.br \\ Recebido para publicação: 01/11/2011 - Aceito para publicação: 07/06/2013
}

\begin{abstract}
O objetivo do trabalho foi analisar a distribuição diamétrica de um fragmento de Floresta Atlântica localizado na Reserva Biológica Sítio Palmares, Espírito Santo. Em $2.400 \mathrm{~m}^{2}$, foram medidas todas as árvores e palmeiras com DAP igual ou superior a $5 \mathrm{~cm}$. Foram encontrados 1.364 indivíduos extrapolados para hectare, distribuídos em 70 espécies e 33 famílias. O total da população observada apresentou um DAP médio de $14,82 \mathrm{~cm}$ e área basal de $41,12 \mathrm{~m}^{2} \cdot \mathrm{ha}^{-1}$. Para o total da população observada, foram formadas 13 classes diamétricas, com intervalo de $5 \mathrm{~cm}$ entre as classes. $\mathrm{O}$ ajuste da equação de Meyer mostrou para essa população a tendência da curva do J-invertido. As três primeiras classes diamétricas, de 5 a $20 \mathrm{~cm}$, concentraram $79 \%$ da densidade populacional. Oito espécies apresentaram uma densidade populacional com mais de 40 indivíduos por hectare. Esses indivíduos ficaram concentrados até a classe diamétrica de $40 \mathrm{~cm}$. A análise estatística mostrou que as curvas de tendências da distribuição diamétrica dessas espécies ficaram distribuídas entre a normal, a exponencial negativa e a bimodal.
\end{abstract}

Resumo

Palavras-chave: Levantamento florístico; classes diamétricas; dendrometria.

\begin{abstract}
Diameter distribution of an Atlantic Forest fragment at Santa Maria de Jetibá county, ES. This research aims to analyse the diametric distribution of an Atlantic Forest fragment at Reserva Biológica Sítio Palmares, state of Espírito Santo. In 2,400 $\mathrm{m}^{2}$ all trees and palms with DBH over $5 \mathrm{~cm}$ were measured. It identified 1364 individuals distributed into 70 species and 33 families. The community population had a DBH average of $14.82 \mathrm{~cm}$ and a basal area of $41.12 \mathrm{~m}^{2} . \mathrm{ha}^{-1}$. For the total population, the DBH distribution into $5 \mathrm{~cm}$ intervals formed 13 classes. The adjusted Meyer's equation reveals for this population a tendency to a J-inverse curve. The first three diameter classes from 5 to $20 \mathrm{~cm}$ concentrated $79 \%$ of the total population. Eight species had a population density with more than 40 trees per hectare. These trees were concentrated until the $40 \mathrm{~cm}$ distribution classes. The statistical analysis of the distributions curves reveals that these populations were distributed between the normal, j-inverse and bi-modal curves.

Keywords: Floristic survey; diametric classes; forest mensuration.
\end{abstract}

\section{INTRODUÇÃO}

As várias fitofisionomias da Floresta Atlântica, inseridas no Bioma Costeiro ou Atlântico, ficaram intimamente relacionadas a fatores tanto naturais como antrópicos, que há pouco mais de cinco séculos se estendiam por toda a costa atlântica brasileira, sendo que atualmente estão restritas principalmente a áreas serranas costeiras das regiões Sul e Sudeste do Brasil (SIMÕES; LINO, 2002; LOUREIRO, 2008; DELDUQUE et al., 2008). Atualmente, os remanescentes da vegetação nativa da Mata Atlântica estão reduzidos em torno de $22 \%$ de sua cobertura original e encontram-se em diferentes 
estágios de crescimento. Registra-se que 7,3\% dessa superfície estão bem preservados, em fragmentos de 100 ha (GUEDES; SEEHUSEN, 2012). Nessas circunstâncias, estima-se que na Mata Atlântica existam mais de 20.000 espécies vegetais (PINTO et al., 2006), correspondendo aproximadamente a $35 \%$ das espécies existentes no Brasil.

A distribuição diamétrica pode ser definida como a caracterização do número de árvores por unidade de área e por intervalo de classe diamétrica, permitindo a aferição do conhecimento da dinâmica e estrutura populacional que compõem a comunidade vegetal a ser observada (ALVES JÚNIOR et al., 2009; SANTANA, 2009). Assim, a distribuição diamétrica torna-se uma eficiente ferramenta para a elaboração de correspondentes planos de ordenação silvicultural e manejo florestal, além de permitir inferências sobre o comportamento do crescimento do maciço florestal.

O presente estudo teve por objetivo analisar a distribuição diamétrica do componente arbóreo de um fragmento de floresta atlântica, em área preservada localizada na Reserva Biológica Sítio Palmares, município de Santa Maria de Jetibá, estado do Espírito Santo, a fim de oferecer subsídios para sua manutenção e possível correspondente plano de manejo florestal. Pretendeu-se verificar como hipótese a possibilidade de estruturar uma sólida e consistente distribuição diamétrica com apenas seis parcelas, totalizando $2.400,00 \mathrm{~m}^{2}$ de área amostral.

\section{MATERIAL E MÉTODOS}

A área de estudo localiza-se na Reserva Biológica Sítio Palmares, no Distrito Rio Claro, do município de Santa Maria de Jetibá, estado do Espírito Santo, em uma altitude de $692 \mathrm{~m}$, região pertencente à área serrana da Mata Atlântica.

O clima da região, segundo a classificação de Köppen, é do tipo tropical de altitude $\mathrm{Cwb}$, com as estações chuvosas e secas bem definidas. A média anual da temperatura é de $18{ }^{\circ} \mathrm{C}$, e a precipitação média anual é de $1.800 \mathrm{~mm}$.

Na Reserva Biológica Sítio Palmares encontram-se áreas preservadas da formação vegetal da Mata Atlântica. Para o presente estudo, foi escolhida aleatoriamente uma dessas áreas, na qual foram instaladas seis parcelas de $400 \mathrm{~m}^{2}(20$ × $20 \mathrm{~m})$ distribuídas aleatoriamente.

Todos os indivíduos arbóreos, incluídas as palmeiras, com Diâmetro à Altura do Peito (DAP) igual ou superior a $5 \mathrm{~cm}$ foram identificados e medidos. A identificação das espécies foi realizada in loco, por um especialista em dendrologia. Para alguns indivíduos, prepararam-se exsicatas, que foram incorporadas ao acervo do herbário da Universidade de Brasília (UB). A sinonímia e a grafia dos taxa foram confirmadas e atualizadas mediante consulta ao índice de espécies do Royal Botanic Garden e do banco de dados do Missouri Botanical Garden (TROPICOS, 2010), exceto Leguminosae, que se considerou como famílias - Fabaceae, Caesalpiniaceae e Mimosaceae -, tendo em vista que as características de cada uma delas são infinitamente distintas no contexto taxonômico.

De cada indivíduo arbóreo foi medido o DAP, com uma suta metálica. A área basal (AB) foi determinada pelo somatório do produto das áreas transversais, considerando a expressão DAP ${ }^{2} \cdot 0,7854$. As áreas basais por classes diamétricas foram calculadas pelo quociente " $q$ " De Liocourt (MEYER, 1952), obtido pela divisão da área basal de uma classe diamétrica pela correspondente da classe anterior, a fim de representar o índice do balanceamento do recrutamento de indivíduos arbóreos.

Para verificar o padrão de distribuição dos DAPs por espécie, o ajuste do número de indivíduos arbóreos por classes diamétricas foi testado e analisado pelos seguintes modelos matemáticos: distribuição exponencial negativa (MEYER, 1952), distribuição normal de Gaussian e distribuição bimodal Waveform (ZAR, 1996). Para a seleção dos modelos ajustados, foi considerado o coeficiente de determinação $\left(\mathrm{R}^{2}\right)$, o erro de ajuste do modelo $(\varepsilon)$ e o nível de significância $(p)$ estatística das equações.

\section{RESULTADOS E DISCUSSÃO}

O levantamento florístico da área estudada constatou a existência de 70 espécies, entre árvores e palmeiras, das quais 68 são arbóreas, distribuídas em 32 famílias, conforme a tabela 1. Duas espécies (Euterpe edulis Mart. e Syagrus botryophora (Mart.) Mart. pertencem à família das palmeiras, Arecaceae). O total da densidade populacional extrapolada para um hectare foi de 1.364 indivíduos com $\mathrm{DAP} \geq 5 \mathrm{~cm}$, incluindo as palmeiras. Excluindo as palmeiras, a população arbórea ficou composta de 
1.104 indivíduos, valor plenamente aceitável, uma vez que ficou enquadrada nas informações registradas na literatura consultada (Tabela 2) para fragmentos de Mata Atlântica.

Tabela 1. Número de indivíduos e área basal por espécie na formação da Mata Atlântica da Reserva Biológica Sítio Palmares.

Table 1. Number of trees and basal area per species of the Atlantic Forest site at the Reserva Biológica Sítio Palmares.

\begin{tabular}{|c|c|c|c|c|}
\hline Família/espécie & $\begin{array}{c}\text { Densidade } \\
\text { extrapolada } \\
\left(\text { n.ha }^{-1}\right)\end{array}$ & $\begin{array}{l}\text { DAP } \\
\text { médio } \\
(\mathbf{c m})\end{array}$ & $\begin{array}{l}\text { Amplitude } \\
\text { diamétrica } \\
(\mathrm{cm})\end{array}$ & $\begin{array}{c}\text { Área } \\
\text { basal }\left(\mathbf{m}^{2} \cdot \text { ha }^{-1}\right)\end{array}$ \\
\hline \multicolumn{5}{|l|}{ ANACARDIACEAE } \\
\hline $\begin{array}{l}\text { Tapirira guianensis Aubl. } \\
\text { ANNONACEAE }\end{array}$ & 32 & 24,8 & $5,5-46,0$ & 2,0816 \\
\hline Xylopia sericea A. St.-Hil. & 52 & 8,8 & $5,0-19,5$ & 0,3967 \\
\hline \multicolumn{5}{|l|}{ APOCYNACEAE } \\
\hline Aspidosperma australe Mull. Arg. & 28 & 24,4 & $9,5-62,0$ & 1,8063 \\
\hline Aspidosperma cylindrocarpon Mull. Arg. & 4 & 30,0 & & 0,2940 \\
\hline Aspidosperma polyneurum Mull. Arg. & 20 & 11,2 & $6,0-21,0$ & 0,2672 \\
\hline Aspidosperma populifolium A. DC. & 4 & 17,0 & & 0,0944 \\
\hline $\begin{array}{l}\text { Aspidosperma subincanum Mart. ex A. DC. } \\
\text { ARECACEAE (Palmae) }\end{array}$ & 8 & 9,5 & $9,0-10,0$ & 0,0591 \\
\hline Euterpe edulis Mart. & 248 & 8,5 & $5,5-12,0$ & 1,5311 \\
\hline \multicolumn{5}{|l|}{ BIGNONIACEAE } \\
\hline $\begin{array}{l}\text { Tabebuia cassinoides (Lam.) DC. } \\
\text { BORAGINACEAE }\end{array}$ & \multicolumn{3}{|c|}{ BORAGINACEAE } & 0,1440 \\
\hline Cordia sellowiana Cham. & 12 & 19,5 & $13,0-31,5$ & 0,4434 \\
\hline \multicolumn{4}{|l|}{ BURSERACEAE } & 0,3532 \\
\hline \multicolumn{5}{|l|}{ CAESALPINIACEAE } \\
\hline Apuleia leiocarpa (Vogel) J. F. Macbr. & 8 & 23,2 & $19,5-27,0$ & 0,3624 \\
\hline Cassia ferruginea (Schrader) Schrader ex DC. & 4 & 13,0 & & 0,0552 \\
\hline Cenostigma angustifolum Tul. & 12 & 18,6 & $14,0-23,0$ & 0,3548 \\
\hline Hymenaea courbaril $\mathrm{L}$. & 12 & 27,6 & $21,5-35,5$ & 0,7836 \\
\hline \multicolumn{5}{|l|}{ CALOPHYLLACEAE (Guttiferae) } \\
\hline \multicolumn{4}{|l|}{ CHRYSOBALANACEAE } & 0,1373 \\
\hline \multirow{2}{*}{\multicolumn{5}{|c|}{ CLUSIACEAE (Guttiferae) }} \\
\hline & & & & \\
\hline Rheedia brasiliensis (Mart.) Planch. \& Triana & 4 & 7,0 & & 0,0160 \\
\hline Symphonia globulifera L. f. & 4 & 26,5 & & 0,2294 \\
\hline \multicolumn{5}{|l|}{ EUPHORBIACEAE } \\
\hline Alchornea triplinervia (Spreng) Mull. Arg. & 4 & 7,5 & & 0,0183 \\
\hline \multicolumn{4}{|l|}{ FABACEAE } & 2,0009 \\
\hline Andira fraxinifolia Benth. & 8 & 15,2 & $5,5-25,0$ & 0,2123 \\
\hline Dalbergia decipularis Rizzini \& A. Mattos & 4 & 47,0 & & 0,7217 \\
\hline Dalbergia nigra (Vell.) Allemao ex Benth. & 16 & 12,8 & $6,5-20,0$ & 0,2524 \\
\hline Ormosia arborea (Vell.) Harms & 4 & 8,0 & & 0,0209 \\
\hline Ormosia fastigiata Tul. & 8 & 18,2 & $17,5-19,0$ & 0,2180 \\
\hline Platycyamus regnellii Benth. & 4 & 27,0 & & 0,2381 \\
\hline Sweetia fruticosa Spreng. & 8 & 37,0 & $17,0-57,0$ & 1,1559 \\
\hline
\end{tabular}




\begin{tabular}{|c|c|c|c|c|}
\hline \multirow{2}{*}{$\begin{array}{l}\text { Vataireopsis araroba (Aguiar) Ducke } \\
\text { HIPPOCRATEACEAE }\end{array}$} & \multirow[t]{2}{*}{4} & \multicolumn{2}{|l|}{16,0} & \multirow[t]{2}{*}{0,0836} \\
\hline & & & & \\
\hline Salacia amygdalina Peyr. & 80 & 9,2 & $5,0-21,0$ & 0,6860 \\
\hline LACISTEMATACEAE & & & & \\
\hline $\begin{array}{l}\text { Lacistema pubescens Mart. } \\
\text { LAURACEAE }\end{array}$ & 12 & 8,0 & $6,0-23,0$ & 0,2539 \\
\hline Mezilaurus navalium (Allemão) Taub. ex Mez & 12 & 12,8 & $6,5-20,0$ & 0,1915 \\
\hline Nectandra myriantha Meisn. & 12 & 9,6 & $7,0-14,0$ & 0,1009 \\
\hline Nectandra reticulata (Ruiz \& Pav.) Mez & 8 & 9,5 & $8,0-11,0$ & 0,0604 \\
\hline Nectandra rigida (Kunth) Nees & 4 & 26,0 & & 0,2208 \\
\hline Ocotea catharinensis Mez. & 52 & 10,0 & $5,0-18,0$ & 0,4902 \\
\hline Ocotea elegans Mez. & 68 & 12,8 & $5,5-31,0$ & 1,2796 \\
\hline Ocotea glomerata (Nees) Mez. & 32 & 21,1 & $6,5-36,0$ & 1,4067 \\
\hline Ocotea pretiosa (Nees) Mez. & 60 & 14,1 & $7,0-23,0$ & 1,2142 \\
\hline Ocotea pulchella Mart. & 24 & 15,3 & $8,0-30,0$ & 0,6235 \\
\hline LECYTHIDACEAE & & & & \\
\hline $\begin{array}{l}\text { Cariniana estrellensis (Raddi) Kuntze } \\
\text { MALVACEAE }\end{array}$ & 12 & 26,0 & $7,0-38,0$ & 0,8436 \\
\hline $\begin{array}{l}\text { Basiloxylon brasiliensis (Allemão) K. Schum } \\
\text { MELASTOMATACEAE }\end{array}$ & 48 & 20,0 & $5,0-33,5$ & 1,9962 \\
\hline Huberia glazioviana Cong. & 4 & 5,7 & & 0,0216 \\
\hline Miconia cinnamomifolia (DC.) Naudin & 16 & 10,2 & $5,5-20,0$ & 0,1707 \\
\hline Miconia minutiflora (Bonpl.) DC. & 28 & 9,0 & $5,0-16,0$ & 0,2203 \\
\hline Cabralea canjerana (Vell.) Mart. & 4 & 10,0 & & 0,0326 \\
\hline $\begin{array}{l}\text { Guarea guidonia (L.) Sleumer } \\
\text { MIMOSACEAE }\end{array}$ & 20 & 16,3 & $8,0-24,0$ & 0,4940 \\
\hline $\begin{array}{l}\text { MIMOSACEAE } \\
\text { Anadenanthera macracarna (Renth ) Brenan }\end{array}$ & & & & \\
\hline Anadenanthera macrocarpa (Benth.) Brenan & 4 & 22,5 & & 0,1654 \\
\hline Enterolobium contortisiliquum (Vell.) Morong & 4 & 31,0 & & 0,3139 \\
\hline Parkia pendula (Willd.) Benth. ex Walp. & 4 & 95,0 & & 2,9487 \\
\hline $\begin{array}{l}\text { Piptadenia communis Benth. } \\
\text { MONIMIACEAE }\end{array}$ & 4 & 14,5 & & 0,0686 \\
\hline $\begin{array}{l}\text { Mollinedia gilgiana Perkins } \\
\text { MORACEAE }\end{array}$ & 4 & 15,5 & & 0,0784 \\
\hline $\begin{array}{l}\text { Ficus enormis (Mart. ex Miq.) Mart. } \\
\text { MYRTACEAE }\end{array}$ & 4 & 18,5 & & 0,1118 \\
\hline Eugenia involucrata DC. & 36 & 13,7 & $5,5-33,0$ & 0,7272 \\
\hline $\begin{array}{l}\text { Myrcia pubipetala Miq. } \\
\text { OPILIACEAE }\end{array}$ & 28 & 7,9 & $6,5-13,0$ & 0,1551 \\
\hline $\begin{array}{l}\text { Agonandra brasiliensis Miers. Ex Benth. \& Hook. F. } \\
\text { PHYTOLACCACEAE }\end{array}$ & 4 & 13,0 & & 0,0552 \\
\hline $\begin{array}{l}\text { Gallesia gorazema (Vel.) Moq. } \\
\text { RUBIACEAE }\end{array}$ & 4 & 13,5 & & 0,0595 \\
\hline Coussarea friburgensis M. Gomes & 12 & 10,5 & $6,5-15,5$ & 0,1217 \\
\hline $\begin{array}{l}\text { Psychotria vellosiana Benth. } \\
\text { SAPINDACEAE }\end{array}$ & 8 & 7,0 & $6,0-8,0$ & 0,0326 \\
\hline $\begin{array}{l}\text { Cupania vernalis Cambess. } \\
\text { SAPOTACEAE }\end{array}$ & 8 & 20,5 & $9,5-31,5$ & 0,3536 \\
\hline Manilkara elata (Allemão ex Miq.) Monach. & 12 & 22,5 & $12,5-40,0$ & 0,6473 \\
\hline Manilkara longifolia (A. DC.) Dubard & 32 & 31,2 & $6,0-13,0$ & 6,4068 \\
\hline $\begin{array}{l}\text { Manilkara rufula (Miq.) H. J. Lam } \\
\text { TILIACEAE }\end{array}$ & 16 & 33,9 & $8,0-95,0$ & 3,4062 \\
\hline $\begin{array}{l}\text { Apeiba tibourbon Aubl. } \\
\text { URTICACEAE (Moraceae) }\end{array}$ & 4 & 30,0 & & 0,2940 \\
\hline
\end{tabular}




\begin{tabular}{lcccc}
\hline Cecropia catarinensis Cautrec. & 4 & 19,0 & & 0,1179 \\
VERBENACEAE & & & & \\
$\begin{array}{l}\text { Aegiphila sellowiana } \text { Cham. } \\
\text { VOCHYSIACEAE }\end{array}$ & 8 & 11,5 & $11,0-12,0$ & 0,0865 \\
Vochysia bifalcata Warm. & 4 & 14,0 & & 0,0640 \\
\hline Total geral & 1.364 & & $5,0-118,0$ & 42,8419 \\
\hline Excluindo as palmeiras & 1.104 & & & 41,1275 \\
\hline
\end{tabular}

Do total das espécies, 28 (40\%) apresentaram menos de cinco indivíduos arbóreos por hectare, representando $8,2 \%$ do total da densidade populacional. Oito espécies - Basiloxylon brasiliensis (Allemão) K. Schum., Euterpe edulis Mart., Maprounea guianensis Aubl., Ocotea catharinensis Mez., Ocotea elegans Mez., Ocotea pretiosa (Nees) Mez., Salacia amygdalina Peyr. e Xylopia sericea A. St.Hil. - apresentaram mais de 40 indivíduos por hectare cada uma. O DAP médio ponderado dessas espécies ficou enquadrado na amplitude de 11,2 a 15,8 $\mathrm{cm}$ (Tabela 3), demonstrando que essa comunidade ainda está em estádio inicial de sucessão secundária, uma vez que indivíduos arbóreos adultos dessas espécies nesse bioma poderão atingir DAP superior a $85 \mathrm{~cm}$ (MAGNANINI; MAGNANINI, 2002). Outra característica marcante se observa na presença da palmeira Euterpe edulis, indicativa do estrato intermediário da Floresta Ombrófila Densa (PÉLLICO NETTO, 1984; MANTOVANI et al., 2005). E. edulis apresentou no presente estudo uma densidade de 248 indivíduos por hectare, valor superior ao recomendado para correspondentes sistemas de manejo e exploração do palmito nas fitofisionomias costeiras da Mata Atlântica (MAFEI, 2004). No entanto, pelo número de indivíduos, a espécie encontra-se na fase imatura da sua correspondente pirâmide demográfica (MANTOVANI et al., 2005). No inventário florestal realizado no estado do Paraná (PÉLLICO NETTO, 1984), essa palmeira ocupou $43 \%$ da abundância relativa da regeneração natural, não tendo sido registrada no estrato arbóreo, podendo ser considerada como pioneira da sucessão natural ecológica silvicultural. Segundo Silva e Leitão Filho (1982) e Guedes-Bruni et al. (1997), E. edulis se apresenta abundantemente em quase todas as associações da Mata Atlântica, tanto em comunidades arbóreas em bom estado de conservação como em matas perturbadas ou secundárias. A espécie Ocotea catharinensis, pelos registros encontrados por Reis et al. (1999), pertence ao grupo de espécies da fase final da sucessão vegetal. Outra observação é que as espécies Salacia amygdalina e Xylopia sericea são típicas no bioma Cerrado. Essas observações sugerem que o sítio estudado faz parte provavelmente de um ecótono.

Tabela 2. Densidade arbórea de algumas áreas da Mata Atlântica.

Table 2. Tree density of some sites of the Atlantic Forest.

\begin{tabular}{lcccc}
\hline Local & $\begin{array}{c}\text { Número } \\
\text { famílias }\end{array}$ & $\begin{array}{c}\text { Número } \\
\text { espécies }\end{array}$ & $\begin{array}{c}\text { Densidade } \\
\left(\mathbf{n . h a}^{-1}\right)\end{array}$ & Autores \\
\hline Recife, PE - região metropolitana & 25 & 48 & 1.268 & Silva et al. $(2009)$ \\
Viçosa, MG - Mata da Pedreira & 30 & 91 & 957 & Marangon et al. $(2008)$ \\
Médio Rio Doce, MG & 23 & 43 & 1.247 & Drumond; Meira Neto (1999) \\
Recife, PE - região metropolitana & 25 & 54 & 1.657 & Alves Júnior et al. $(2007)$ \\
Reserva Biológica Sítio Palmares, ES & 32 & 68 & 1.104 & Este estudo \\
\hline
\end{tabular}

O DAP médio aritmético do total da população de indivíduos arbóreos foi de $14,8 \mathrm{~cm}$. Pelo valor do DAP médio, pode-se inferir que a comunidade vegetal observada atualmente pertence silviculturalmente a uma estrutura arbórea de porte médio ainda em processo inicial de crescimento da sucessão natural.

Distribuídos os DAPs do total da população, em classes de diâmetro com $5 \mathrm{~cm}$ de intervalo (Figura 1), verificou-se que foram estabelecidas 13 classes diamétricas, na amplitude de 5 a $120 \mathrm{~cm}$. A curva de distribuição de Meyer mostrou a típica tendência do $\mathrm{J}$ invertido, expressa pela equação ajustada $y=416,6597 \cdot e^{(-0,1326 x)}$. Ela apresentou um coeficiente de determinação $\left(\mathrm{R}^{2}\right)$ igual a 0,98 , com um erro $(\varepsilon)$ de modelo de 0,012 e um nível de significância $(p)<0,001$. Portanto, pode-se considerar a equação significativa para a população observada. 
Pode-se verificar que as classes diamétricas não acompanharam plenamente a curva traçada (Figura 1), podendo-se inferir que nas classes diamétricas acima de $35 \mathrm{~cm}$ devem ter existido ações antrópicas ou naturais que diminuíram o número de indivíduos, apresentando déficit nessas classes. Esse fato pode ser ainda comprovado pelo quociente De Liocourt “ $q$ " (MEYER, 1952) calculado na Tabela 3 , quando se demonstra que a vegetação arbórea observada não se encontra balanceada, uma vez que os valores obtidos ficaram diferentes e irregulares entre si.

As interrupções dos histogramas de frequência nas diversas classes diamétricas mostraram que os ciclos de vida natural das espécies ainda não se estabilizaram no modelo de crescimento clímax. Outra característica marcante da estrutura diamétrica (Figura 1) é que as três primeiras classes diamétricas, na amplitude de 5 a $20 \mathrm{~cm}$, concentraram 79,5\% da densidade populacional (Tabela 3).

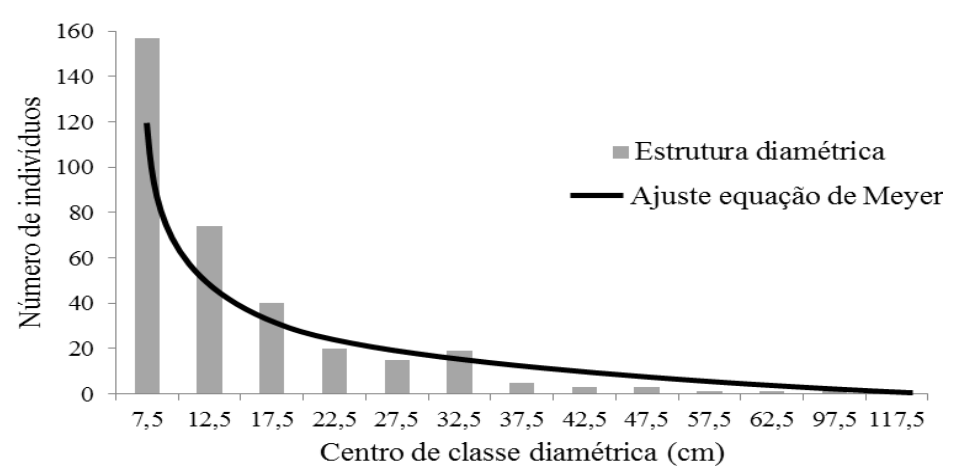

Figura 1. Curva de tendência do J invertido na distribuição diamétrica do total da população, extrapolado para o hectare.

Figure 1. J inverse curve in diametric distribution of the total population, extrapolated for hectare.

A curva da distribuição do $J$ invertido ou exponencial negativo (Figura 1) sugere, para o caso da população medida, que a densidade populacional está em estado incompleto de tendência à autorregeneração, principalmente nas primeiras classes diamétricas, onde não existe o balanço natural entre a mortalidade e o recrutamento dos indivíduos pertinentes em cada uma dessas classes, corroborado pelo quociente De Liocourt (Tabela 3). As classes de 40, 45, 55 e 60 encontram-se com taxas de recrutamento e mortalidade balanceadas.

Tabela 3. Distribuição arbórea (sem as palmeiras) em classes diamétricas de $5 \mathrm{~cm}$ de intervalo.

Table 3. Trees distribution (without palms) in DBH classes of $5 \mathrm{~cm}$ interval.

\begin{tabular}{|c|c|c|c|c|c|c|}
\hline \multirow[b]{2}{*}{$\begin{array}{l}\text { Classe diamétrica } \\
(\mathbf{c m})\end{array}$} & \multirow[b]{2}{*}{$\begin{array}{l}\text { CCD } \\
\text { (cm) }\end{array}$} & \multicolumn{2}{|c|}{ Densidade } & \multicolumn{2}{|c|}{ Área basal } & \multirow[b]{2}{*}{ "q" } \\
\hline & & Absoluta & $\begin{array}{c}\text { Relativa } \\
(\%)\end{array}$ & $\begin{array}{c}\text { Total } \\
\left(\mathbf{m}^{2}\right)\end{array}$ & $\begin{array}{r}\text { Acumulada } \\
(\%)\end{array}$ & \\
\hline $5,0-10,0$ & 7,5 & 628 & 46,04 & 2,7327 & 6,6 & 0,12 \\
\hline $10,0-15,0$ & 12,5 & 296 & 21,70 & 3,2982 & 14,6 & 1,11 \\
\hline $15,0-20,0$ & 17,5 & 160 & 11,73 & 3,6590 & 23,4 & 0,80 \\
\hline $20,0-25,0$ & 22,5 & 80 & 5,87 & 2,9473 & 30,5 & 1,08 \\
\hline $25,0-30,0$ & 27,5 & 60 & 4,40 & 3,1979 & 38,2 & 1,87 \\
\hline $30,0-35,0$ & 32,5 & 76 & 5,57 & 5,9703 & 52,6 & 0,45 \\
\hline $35,0-40,0$ & 37,5 & 20 & 1,47 & 2,7106 & 59,1 & 0,60 \\
\hline $40,0-45,0$ & 42,5 & 12 & 0,88 & 1,6377 & 63,0 & 1,27 \\
\hline $45,0-50,0$ & 47,5 & 12 & 0,88 & 2,0825 & 68,0 & 0,49 \\
\hline $55,0-60,0$ & 57,5 & 4 & 0,29 & 1,0207 & 70,4 & 1,18 \\
\hline $60,0-65,0$ & 62,5 & 4 & 0,29 & 1,2076 & 73,3 & 5,26 \\
\hline $95,0-100,0$ & 97,5 & 8 & 0,59 & 6,3548 & 88,7 & 0,68 \\
\hline $115,0-120,0$ & 117,5 & 4 & 0,29 & 4,3743 & 100,0 & \\
\hline Total & & 1364 & 100 & 41,1936 & & \\
\hline
\end{tabular}

CCD: centro da classe diamétrica; "q": quociente De Liocourt. 
Em relação à área basal, excluídas as palmeiras, foi encontrada uma superfície de ocupação de 41,12 $\mathrm{m}^{2} \cdot \mathrm{ha}^{-1}$ (Tabela 3). O equilíbrio natural da área basal, na vegetação estudada, se deu na classe diamétrica 30 - $35 \mathrm{~cm}$, uma vez que $50 \%$ da área basal estão contidas até essa classe diamétrica.

Braga et al. (2009) registraram a existência em um fragmento de Mata Atlântica localizado na Reserva Florestal e Ecológica Mata do Paraíso, em Viçosa (MG), de 21,27 a 60,35 $\mathrm{m}^{2}$.ha ${ }^{-1}$. Oliveira (2002) encontrou 26,3 e 32,4 $\mathrm{m}^{2} \cdot \mathrm{ha}^{-1}$ para áreas de 25 e 50 anos de idade de um fragmento litorâneo da Mata Atlântica em Ilha Grande (RJ), respectivamente. Drumond e Meira Neto (1999) registraram, após 25 anos de regeneração natural, na região do Médio Rio Doce (MG), uma área basal igual a $16,7 \mathrm{~m}^{2} \cdot \mathrm{ha}^{-1}$. Alves Júnior et al. (2007), em estudo realizado nos fragmentos da Mata Atlântica na Região Metropolitana de Recife (PE), encontraram área basal entre 24,70 e 41,68 $\mathrm{m}^{2} \cdot \mathrm{ha}^{-1}$. Demonstra-se, assim, que os valores mencionados corroboram área basal encontrada neste estudo.

Das 70 espécies, a Manilkara longifolia, com 32 indivíduos arbóreos, apresentou a maior área basal, correspondendo a 6,4 $\mathrm{m}^{2} \cdot \mathrm{ha}^{-1}$. Seguiram, na ordem decrescente, com 3,40 e 2,08 $\mathrm{m}^{2} \cdot \mathrm{ha}^{-1}$, respectivamente, as espécies Manilkara rufula, com 16 árvores, e Tapira guianensis, com 32 espécimes. Essas três espécies perfizeram $27 \%$ do total ocupado pela área basal. As três espécies com maior número de indivíduos foram Euterpe edulis, Maprounea guianensis e Salacia amygdalina, que totalizaram 32\% da densidade populacional, ocupando 4,2180 $\mathrm{m}^{2} \cdot \mathrm{ha}^{-1}$, correspondente a $10,2 \%$ do total da área basimétrica.

Nas oito espécies que apresentaram o maior número de indivíduos arbóreos - Basiloxylon brasiliensis (48 árvores), Euterpe edulis (248 árvores), Maprounea guianensis (112 árvores), Ocotea catharinensis (52 árvores), Ocotea elegans (68 árvores), Ocotea pretiosa (60 árvores), Salacia amygdalina (80 árvores) e Xylopia sericea (32 árvores) -, foi efetuada a análise correspondente da distribuição diamétrica (Tabela 4), nos mesmos moldes que foram aplicados ao total da densidade populacional. Correspondentes resultados sintetizados são apresentados na figura 2. As amplitudes das distribuições diamétricas se estenderam de 5 a $45 \mathrm{~cm}$, sendo que a maior concentração dos indivíduos arbóreos ficaram nas três primeiras classes, demonstrando assim que as correspondentes populações se orientam a pertencer a comunidades silviculturais ainda em crescimento inicial ao clímax.

Tabela 4. Frequência da distribuição diamétrica das oito espécies com maior número de indivíduos por classe diamétrica.

Table 4. Frequency of the diametric distribution of the eight mayor species per diametric class.

\begin{tabular}{lccccccccc}
\hline $\begin{array}{l}\text { Classe } \\
\text { diamétrica } \\
(\mathbf{c m})\end{array}$ & $\begin{array}{c}\mathbf{C C D} \\
\mathbf{( c m )}\end{array}$ & $\begin{array}{c}\text { Basiloxylon } \\
\text { brasiliensis }\end{array}$ & $\begin{array}{c}\text { Euterpe } \\
\text { edulis }\end{array}$ & $\begin{array}{c}\text { Maprounea } \\
\text { guianensis }\end{array}$ & $\begin{array}{c}\text { Ocotea } \\
\text { catharinensis }\end{array}$ & $\begin{array}{c}\text { Ocotea } \\
\text { elegans }\end{array}$ & $\begin{array}{c}\text { Ocotea } \\
\text { pretiosa }\end{array}$ & $\begin{array}{c}\text { Salacia } \\
\text { amygdalina }\end{array}$ & $\begin{array}{c}\text { Xylopia } \\
\text { sericea }\end{array}$ \\
\hline $5-10$ & 7,5 & 4 & 68 & 48 & 12 & 20 & 12 & 48 & 28 \\
$10-15$ & 12,5 & 16 & 164 & 24 & 32 & 28 & 24 & 16 & 12 \\
$15-20$ & 17,5 & 0 & 16 & 12 & 0 & 8 & 8 & 8 & 8 \\
$20-25$ & 22,5 & 4 & & 16 & 8 & 4 & 8 & 4 & 4 \\
$25-30$ & 27,5 & 4 & & 4 & & 0 & 4 & 4 & \\
$30-35$ & 32,5 & 8 & & 0 & & 4 & 4 & & \\
$35-40$ & 37,5 & 12 & & 0 & & 4 & & & \\
$40-45$ & 42,5 & & & 8 & & & & & \\
\hline Totais & & 48 & 248 & 112 & 52 & 68 & 60 & 80 & 52 \\
\hline DAP & & 13,7 & 11,4 & 15 & 12,8 & 14,8 & 15,8 & 11,2 & 11,3 \\
\hline
\end{tabular}

CCD: centro da classe diamétrica; $\mathrm{DAP}_{\mathrm{mp}}$ : DAP médio ponderado.

Das oito espécies, quatro delas - Maprounea guianensis, Ocotea elegans, Salacia amygdalina e Xylopia sericea - podem estar plenamente asseguradas no processo de recrutamento da regeneração natural, uma vez que as colunas demonstrativas (Figura 2) acompanham a curva da equação exponencial negativa de Meyer. A distribuição diamétrica da espécie Ocotea catharinensis e Ocotea pretiosa mostraram curvas de tendência da distribuição diamétrica para a curva normal de Gaussian, e Basiloxylon brasiliensis apresentou uma distribuição bimodal, mostrando que em algum momento do passado provavelmente essas espécies sofreram uma intervenção antrópica ou perturbação natural. No caso 
específico de Euterpe edulis, pode-se afirmar que no local de estudo ainda existem ações antrópicas de extração da espécie para a produção do palmito. As respectivas estatísticas das equações ajustadas para as oito espécies constam na tabela 5 , onde se pode observar que as curvas traçadas pela equação correspondente podem ser consideradas plenamente representativas para as populações das espécies observadas.
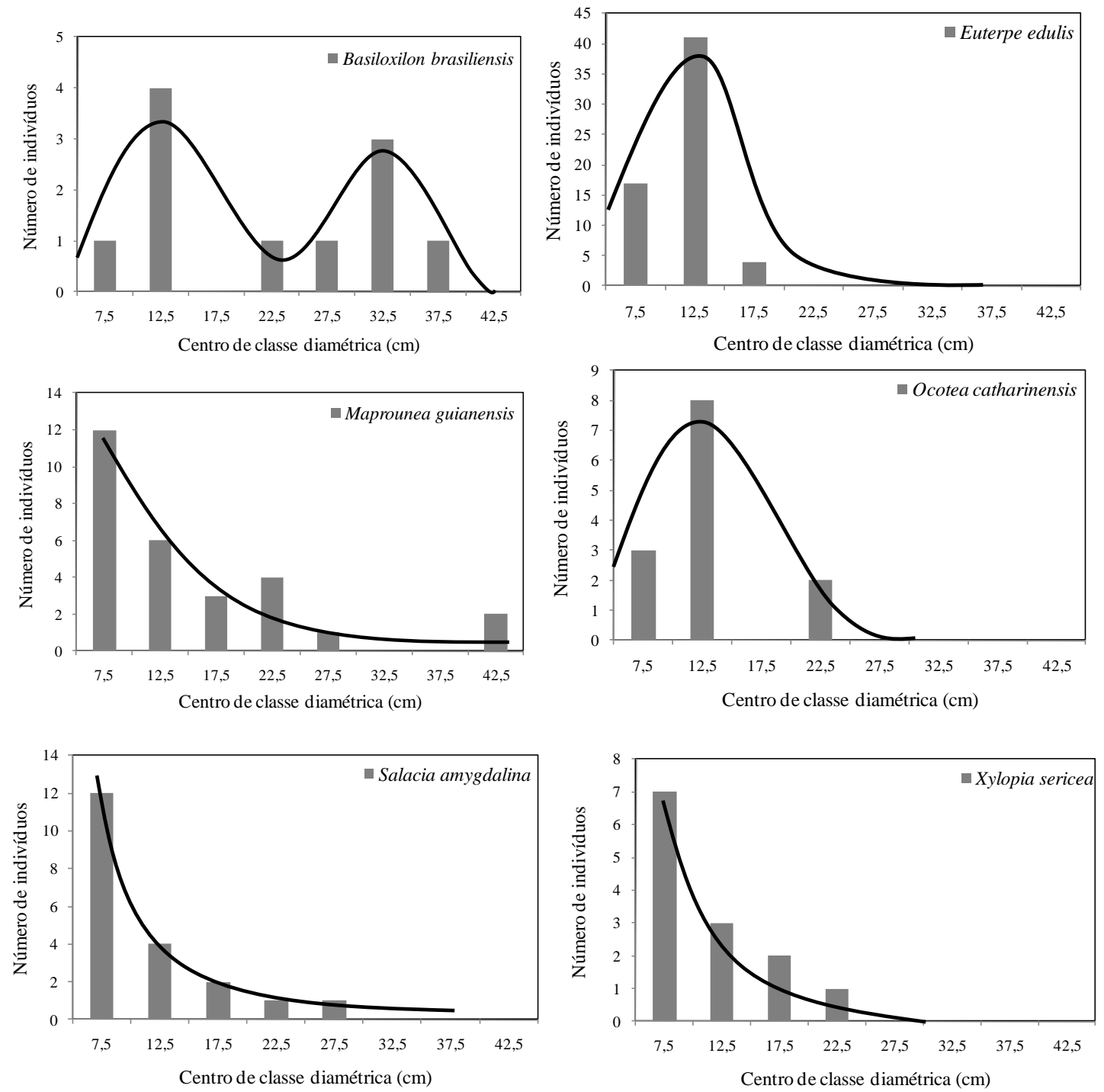

Figura 2. Curvas de tendência da distribuição diamétrica das oito espécies com maior número de indivíduos.

Figure 2. Diametric distribution tendency curves of the eight major species. 
Tabela 5. Estatísticas das equações selecionadas, mostradas na figura 2.

Table 5. Equations statistics for the figure 2.

\begin{tabular}{lcccc}
\hline Espécie & Equação & $\mathbf{R}^{\mathbf{2}}$ & $\boldsymbol{S}_{\boldsymbol{x y}}$ & $\boldsymbol{p}$ \\
\hline Basiloxylon brasiliensis & $y=2,881\left[\sin \left(-\frac{\pi(x+19,581)}{17,212}\right)^{2}\right]$ & 0,83 & 0,123 & $<0,001$ \\
Euterpe edulis & $y=44,490 e^{\left.--0,5\left(\frac{x-1,1370}{2,792}\right)\right]}$ & 0,91 & 0,087 & $<0,001$ \\
Maprounea guianensis & $y=27,172 e^{-0,112 x}$ & 0,95 & 0,101 & 0,034 \\
Ocotea catharinensis & $y=63,261 e^{\left.-0,5\left(\frac{x-1,110}{10,240}\right)\right]}$ & 0,80 & 0,027 & $<0,001$ \\
Ocotea elegans & $y=11,452 e^{-0,008 x}$ & 0,77 & 0,082 & 0,006 \\
Ocotea pretiosa & $y=5,740 e^{\left[-0,5\left(\frac{x-4,262}{12,150}\right)\right]}$ & 0,88 & 0,184 & $<0,001$ \\
Salacia amygdalina & $y=50,481 e^{-0,192 x}$ & 0,97 & 0,025 & $<0,001$ \\
Xylopia sericea & $y=19,690 e^{-0,141 . x}$ & 0,98 & 0,049 & $<0,001$ \\
\hline
\end{tabular}

\section{CONCLUSÕES}

- A distribuição diamétrica do total da densidade populacional observada mostrou acompanhar a típica tendência da curva do $\mathrm{J}$ invertido. As análises diamétricas das oito espécies com maior número de indivíduos apresentaram comportamentos de distribuição dos seus indivíduos fora dos padrões de interpretação dos modelos exponenciais esperados. Comprova-se que foi possível estruturar a consistente distribuição diamétrica em base de relativamente poucas parcelas de amostragem.

- Os recrutamentos naturais dos indivíduos nas diversas classes diamétricas das espécies analisadas mostraram para a área amostrada não estarem assegurados para um crescimento regular ao clímax.

\section{REFERÊNCIAS}

ALVES JÚNIOR, F. T.; FERREIRA, R. L. C.; SILVA, J. A. A. da; MARANGON, L. C.; COSTA JÚNIOR, R. F. Estrutura diamétrica de um fragmento de Floresta Atlântica em matriz de cana-de-açúcar, Catende, Pernambuco. Revista Brasileira de Engenharia Agrícola e Ambiental, v. 13, n. 3, p. 328 333, 2009.

ALVES JÚNIOR, F. T.; SILVA BRANDÃO, C. F. L.; ROCHA, K. D. da; SILVA, J. T. da; MARANGON, L. C.; FERREIRA, R. L. C. Estrutura diamétrica e hipsométrica do componente arbóreo de um fragmento de Mata Atlântica, Recife, PE. Cerne, v. 13, n. 1, p. 83 - 95, 2007.

BRAGA, C. S.; BARBOSA, V. H. B.; GAZZOLA, C. E.; DIAS, H. C. T. Relação entre área basal e a precipitação efetiva em um fragmento de Mata Atlântica na zona da mata norte mineira. In: SEMINÁRIO DE RECURSOS HÍDRICOS DA BACIA DO PARAÍBA DO SUL: RECUPERAÇÃO DE ÁREAS DEGRADADAS, SERVIÇOS AMBIENTAIS E SUSTENTABILIDADE, 2., 2009, Taubaté. Anais do..., 2009. p. 661 - 665.

DELDUQUE, M.; MILANEZ, F.; RIBEIRO, H.; NOGUEIRA-NETO, P. Mata Atlântica. Porto Alegre: Terrabrasil, 2008. 260 p.

DRUMOND, M. A.; MEIRA NETO, J. A. Composições florística e fitossociológica de uma mata secundária de um trecho da Mata Atlântica. Ciência Rural, v. 29, n. 4, p. 657 - 661, 1999.

GUEDES, F. B.; SEEHUSEN, S. E. Pagamentos por serviços ambientais na Mata Atlântica, lições aprendidas e desafios. 2. ed. Brasília: MMA, Secretaria de Biodiversidade e Florestas, 2012. 272 p.

GUEDES-BRUNI, R. R.; PESSOA, S. de V. A.; KURTZ, B. C. Florística e estrutura do componente arbustivo-arbóreo de um trecho preservado de Floresta Montana na Reserva Ecológica de Macaé de Cima. In: LIMA, H. C.; Guedes-Bruni, R. R. Serra de Macaé de Cima: diversidade florística e conservação em Mata Atlântica. Rio de Janeiro, Instituto de Pesquisas Jardim Botânico do Rio de Janeiro, p. $127-145,1997$. 
LOUREIRO, W. RPPN Mata Atlântica: ICMS ecológico, uma experiência brasileira de pagamentos por serviços ambientais. Belo Horizonte: Conservação Internacional, Fundação SOS Mata Atlântica, The Nature Conservancy, 2008. 26 p.

MAFEI, R. A. Considerações ao manejo do palmito (Euterpe edulis Martius). São Paulo: Unicamp, 2004. 81 p. (Série Monografias).

MAGNANINI, A.; MAGNANINI, C. Árvores gigantescas da terra e as maiores assinaladas no Brasil. 2. ed. São Paulo: Conselho Nacional de Reserva de Biofera da Mata Atlântica, 2002. 46 p.

MARANGON, L. C.; SOARES, J. J.; FELICIANO, A. L.; SILVA BRANDÃO, C. F. L. Regeneração natural em um fragmento de floresta estacional semidecidual em Viçosa, Minas Gerais. Árvore, v. 32, n. 1, p. 183 - 191, 2008.

MEYER, H. C. Structure, growth and drain in balanced uneven-aged forests. Journal of Forestry, Washington, DC, v. 50, p. 85 - 92, 1952.

OLIVEIRA, R. R. Ação antrópica e resultantes sobre a estrutura e composição da Mata Atlântica na Ilha Grande, RJ. Rodriguésia, v. 53, n. 82, p. 33 - 58, 2002.

PÉLLICO-NETTO, S. Inventário florestal nacional, florestas nativas: Paraná/Santa Catarina. Brasília: IBDF, 1984. 309 p.

PINTO, L. P.; BEDÊ, L.; PAESE, A.; FONSECA, M.; PAGLIA, A.; LAMAS, I. Mata Atlântica brasileira: os desafios para a conservação da biodiversidade de um hotspot mundial. In: ROCHA, C. F. D.; BERGAllO, H. G.; van SLUYS, M.; ALVES, M. A. S. (Orgs.). Biologia da conservação: essências. San Carlos, Brasil: Rima Artes e Textos, 2006.

REIS, A.; ZAMBONIN, R. M.; NAKAZONO, E. M. Recuperação de áreas florestais degradadas utilizando a sucessão e as interações planta-animal. São Paulo, Reserva da Biosfera da Mata Atlântica - MAB - UNESCO: 1999. 42 p. (Série Recuperação, Caderno n ${ }^{\circ}$ 14)

SANTANA, J. A. da S. Padrão de distribuição e estrutura diamétrica de Croton sonderiamus Muell. Arg. (marmeleiro) na Caatinga da Estação Ecológica do Seridó. Revista Verde, v. 4, n. 3, p. 85 - 90, 2009.

SILVA, A. F.; LEITÃO-FILHO, H. de F. Composição florística e estrutura de um trecho da Mata Atlântica de encosta no Município de Ubatuba (São Paulo, Brasil). Revista Brasileira de Botânica, v. 5, n. p. 42 - 52. 1982.

SILVA, M. A. M.; GUERRA, T. N. F.; MENDES, P. G. de A.; BARBOSA, M. D.; ROCHA, K. D.; CHAVES, L. de F. de C. Diversidade da regeneração natural de diferentes amostragens em um fragmento de floresta atlântica, Recife, PE. In: JORNADA DE ENSINO, PESQUISA E EXTENSÃO, IX, 2009, Recife, UFRPE. Resumos... Recife, 2009. 3 p.

SIMÕES, L. L.; LINO, C. F. Sustentável Mata Atlântica: a exploração de seus recursos florestais. São Paulo: SENAE, 2002. 216 p.

TROPICOS. Disponível em: <www.tropicos.org>. Acessos em: dezembro de 2009 e janeiro de 2010.

ZAR, J. H. Bioestatistical analysis. Upper Saddle River: Prentice Hall, 1996. 662 p. 